\title{
A simple method for collecting airborne pollen
}

\author{
Peter G Kevan ${ }^{1}$, Franco DiGiovanni ${ }^{2}$, Rong H Ho, Hisatomo Taki ${ }^{1}$, Kristyn A Ferguson ${ }^{3}$ \\ and Agata K Pawlowski ${ }^{1}$
}

${ }^{1}$ University of Guelph, ${ }^{2}$ AirZOne Inc, ${ }^{3}$ University of Waterloo, Canada

\begin{abstract}
Pollination is a broad area of study within biology. For many plants, pollen carried by wind is required for successful seed set. Airborne pollen also affects human health. To foster studies of airborne pollen, we introduce a simple device - the 'megastigma' - for collecting pollen from the air. This device is flexible, yielding easily obtained data that can be readily analysed. Thus, it is ideally suited for use in upper level biology courses. Using maize, Zea mays, as our sample study species, we obtained a graph showing daily amounts of pollen in the air. The megastigma can be applied to a wide range of plant species, from herbs to trees, be deployed in a wide variety of ways, and so have potential for many palynological applications.

Key words: Megastigma; Pollen trap; Airborne; Wind; Pollination
\end{abstract}

\section{Introduction}

Pollination is a broad and familiar area in biological education (Claypoole and Slesnick 1983; Aston 1987; Tse and Chan 2001). The process of pollination is mediated by tiny pollen grains, the multicellular haploid male gametophytes produced by seed plants. Pollen grains develop and mature on anthers and are then transferred to stigmata where, if conditions are right, they germinate. Pollen then induces the production of a pollen tube which grows through the style and enters the ovary. There, liberation of the male gametophytes precedes double fertilisation of the cells in the plant's ovary. This process allows the plant to produce seeds; the means of passage for a plant from one generation to the next.

The transfer of pollen may be biotic (mediated by animals) or abiotic (mediated by wind or water). For many economically valuable plants, such as forest and ornamental trees, cereals, and grasses, wind pollination is required in order for the plant to set seed successfully. Issues of pollen dispersal have also been highlighted in regards to potential impacts of genetically-modified crops (e.g. Losey et al, 1999; Barton and D racup 2000; Dale et al, 2002).

It is also noted that windborne pollen can be a major problem for allergy sufferers, to the extent that weather forecasts often include quantitative information on airborne pollen. Chemical components of pollen can aggravate the immune system, resulting in allergies that range from mild hay fever symptoms to serious allergic asthma (e.g. Bhalla et al, 1999; M arquez et al, 2002).

Given its importance both in plant reproduction and in human health, airborne pollen has been studied extensively for its chemical nature, its abundance and diversity, and for seasonal and daily timing of its release. Not surprisingly, numerous gadgets and machines have been developed and used for studies of aeropalynology (Kevan, 2005).

However, the method we present is amongst the simplest and most flexible, making it ideal for use in out door 'classroom' setting. It is both easy and inexpensive to make the pollen traps, referred to as 'megastigmas'. The traps and subsequent pollen collected can be handled easily and analysed without the need for stains. Beyond this, data recording and analyses are also simple. The following explains how to make and use the traps. We also give an example of results obtained from their use in a simple field trial.

\section{Materials and methods}

\section{M egastigma construction}

1. M ake a number of wooden platforms, about $10 \mathrm{~cm}$ square with $45^{\circ}$ bevelled edges and a central hole which can accept a dowel, rod or rope as an axis (Figure 1a).

2. To each bevelled edge of the platform, affix two wooden spring-operated clothes pegs by the non-clip end with weather-resistant glue, nailing or stapling; each platform thus has eight clothes pegs (Figure 1a). Use of water-soluble glue and plastic clothes pegs may prove to be less durable.

3. Thread these platforms onto a chosen axis (length optional) which should fit through holes in the centre of each platform.

4. Make holes across the diameter of the axis to allow for stops, such as nails or toothpicks, to be inserted. These hold the platforms in place. Rope can be used instead of dowel for suspending traps, but it is important that the platforms be close to horizontal.

5. M ake detachable pollen traplets from plastic horticultural 


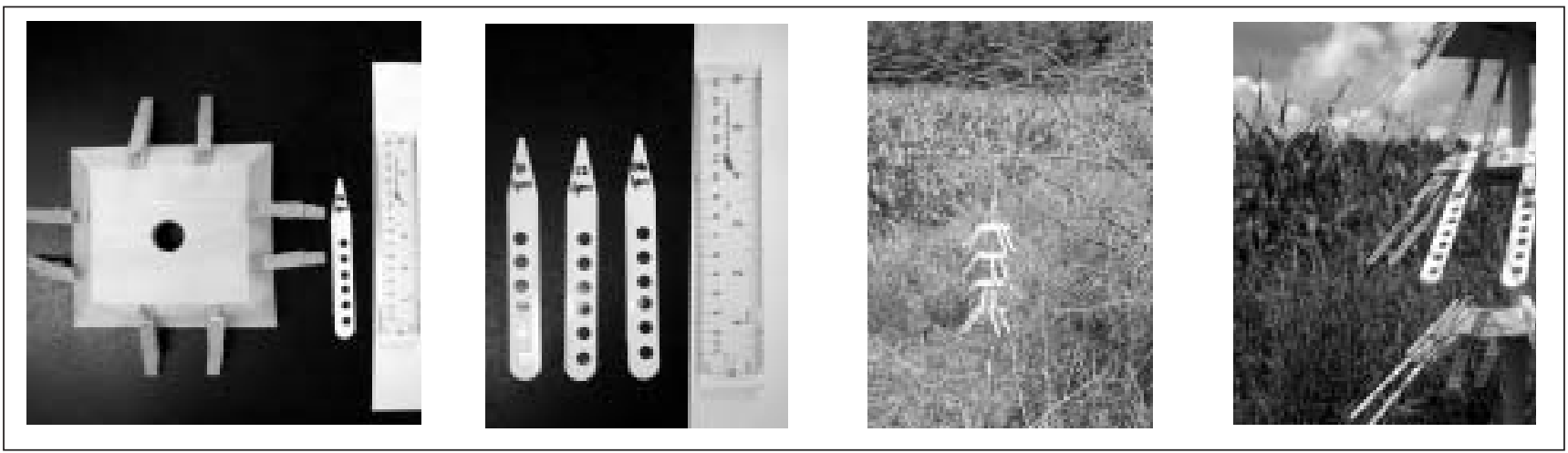

Figure 1 (a-d, left to right). a) O ne platform of megastigma on left with a traplet shown on right. b) Traplets used in current trial. c) and d) M egastigma in operation alongside a cornfield in Guelph, O ntario.

labels, about $1.5 \mathrm{~cm}$ wide $\times 10 \mathrm{~cm}$ long $\times 0.1 \mathrm{~cm}$ thickness (Figure $1 b$ ). U se a single-hole punch to distribute four to six holes evenly down the length of the label.

6. Apply transparent sticky tape along the underside length of the plastic labels so that the sticky surface of the tape is exposed upwards through the holes.

7. Clip the traplets to each platform with the clothes pegs. Each platform should then contain eight traplets with approximately 40 sticky pollen-trapping surfaces in total. If more sampling surfaces are desired, more platforms and/or with more clothes pegs can be constructed.

The platforms can be placed on top of poles, sticks, or dowels which are stuck into, or affixed to, substrates such as soil, sand, posts, or stumps. They can be hoisted into tree canopies or even flown from weather balloons. The whole apparatus should be allowed to swing freely in the air so that it can twist and turn without restriction. This motion allows the traplet array to sample pollen in the air in a more or less random fashion with respect to wind direction. Because of the thickness of the plastic labels, the sticky surfaces are protected in the holes of the labels so that they can be stacked before and after use. Thus, they can be readily packaged, transported, handled and stored.

The design of the megastigma allows for controlled serial collection of traplet samples for variable durations and conditions. It also permits sampling on vertical gradients, from as low as the ground to as high up as weather balloons (e.g. DiG iovanni et al, 1996). The standard size of the holes provides for quantification of the number of grains caught per unit area, and replication for experimental design and statistical analysis.For more in-depth studies, pollen trapped on the sticky surfaces can be stained for easier viewing under the microscope and tested for enzymatic activity or viability (D afni et al, 2005).

\section{Sampling pollen grains}

A ccording to a regimen dictated by the hypothesis to be tested, the traplets can be harvested from the megastigma randomly with respect to position, but systematically with respect to time, height above the ground or canopy, or both. The relative diversity and abundance of pollen caught on the traplets can then be compared.

Recall that the traplets sample pollen from the air that passes over them, so naturally under conditions of high wind more air passes the sticky surface. Therefore, if there are major changes in weather while sampling is being done, pollen abundance can be assessed only qualitatively. This point can be used for discussion with more advanced students on how one might quantitatively sample airborne pollen.

The following protocol, used for grass, is suggested for illustration and simplicity (Kevan, 2005) but can be readily adapted for other plants and conditions.

1. Choose a patch of plants in which to make the study (Figure $1 \mathrm{c}$ and $\mathrm{d}$ ).

2. Early in the morning (dawn) deploy one megastigma with traplets, to which tape has been most recently applied, in place.

3. Record time, height, and weather conditions.

4. A t regular intervals, remove or replace the traplets from the megastigma and return them to the laboratory.

5. Record time, height, and weather conditions at each sampling.

6. Examine each hole in each traplet microscopically at a resolution of 100X or more, counting grains per hole by scanning systematically (Figure 2 ). If necessary, to ease counting, a tiny drop of Calberla's stain (or other stain) ( $D$ afni et al, 2005) can be used in each hole. The stain should be removed after about two minutes by absorbing it with the point of a piece of filter paper or paper towel. Be sure not to dab the hole, but to use only the point of the absorptive surface which allows surface tension to absorb excess stain.

Figure 2. a) Kristyn Ferguson counting pollen grains captured on traplets. b) and c) Microscopic views, 250X, of a single hole from two separate traplets showing Zea mays pollen grains.

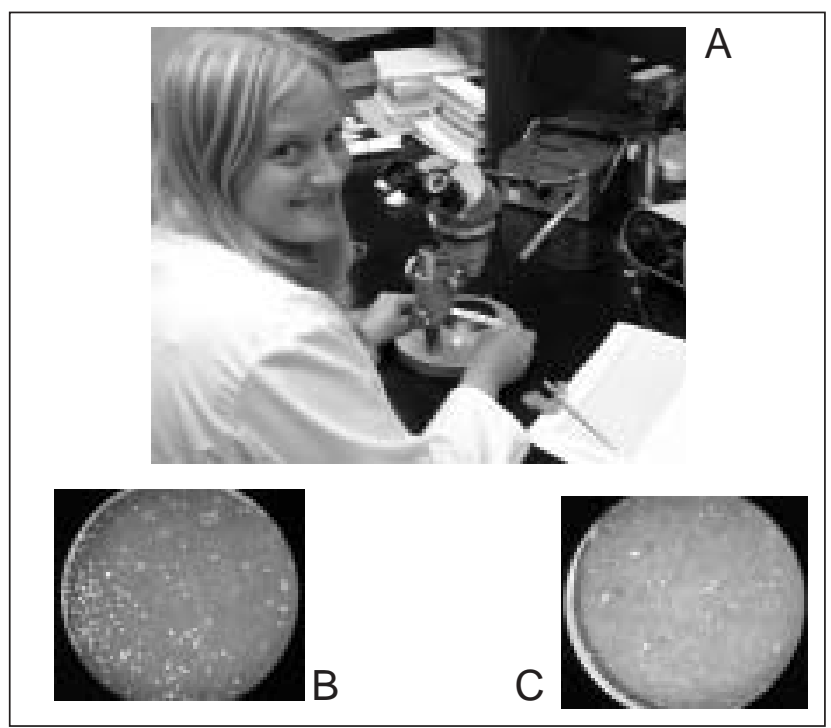


7. Calculate the number of grains per unit area accumulated over the period of exposure to windborne pollen.

8. Plot the results. The trend should show a period of no pollen, then a short lag-phase of accumulation as pollen dispersal starts, then a rapid rise in accumulated pollen, with an asymptote after pollen dispersal ceases or a rapid decline in pollen capture.

Traplets can be deployed at specific times for specific durations and at specific locations for more detailed studies.

\section{A trial with traps}

A simple, day-long experiment was made alongside a field of maize, Zea mays, in Wellington County, O ntario, Canada. A single megastigma trap with 32 , six-hole traplets was stationed at about $2 \mathrm{~m}$ from the periphery of the maize field, where it was hung from a tree branch about $1 \mathrm{~m}$ high, the height at which corn silks (stigmata) occur on the maize plant (Figure 1c and d). The traplets were harvested and replaced every three hours for the 24-hour duration of the experiment, which commenced at 4.00am EDST on 28 July, 2005, and finished at $4.00 \mathrm{am}$ EDST on 29 July, 2005. The average number of maize pollen grains captured on each traplet (combined grain count for all six holes on a given traplet) from 32 traplets sampled at intervals over the trial period is shown in Figure 3.

\section{Results and discussion}

The peak of pollen capture is clearly shown between the time periods of $10.00 \mathrm{am}$ to $1.00 \mathrm{pm}$ and $1.00 \mathrm{pm}$ to $4.00 \mathrm{pm}$ (Figure 3). In this particular illustrative exercise, the day was quite calm (wind speeds about $5 \mathrm{~km} / \mathrm{h}$ ). Maize pollen is mostly shed between one and two hrs after sunrise and in late morning (Snetselaar et al, 2001). D epending on weather conditions, the pollen can remain moist and thus not readily airborne until it dries. Therefore, given a more humid morning the pollen becomes airborne somewhat later in the day. This phenomenon is illustrated by the extended peak of pollen abundance demonstrated in our results. M ore advanced students can explore why periods of maximal pollen capture may not necessarily coincide with periods of maximal airborne pollen content.

\section{Conclusions}

The simplicity of the equipment and methods employed in this experiment make it easily executable for anyone inter-

Figure 3. Trend of pollen flow. M ean \pm SE of number of Zea mays pollen grains captured by 32 traplets of a megastigma at $3 \mathrm{hr}$ time intervals over a $24 \mathrm{hr}$ period.

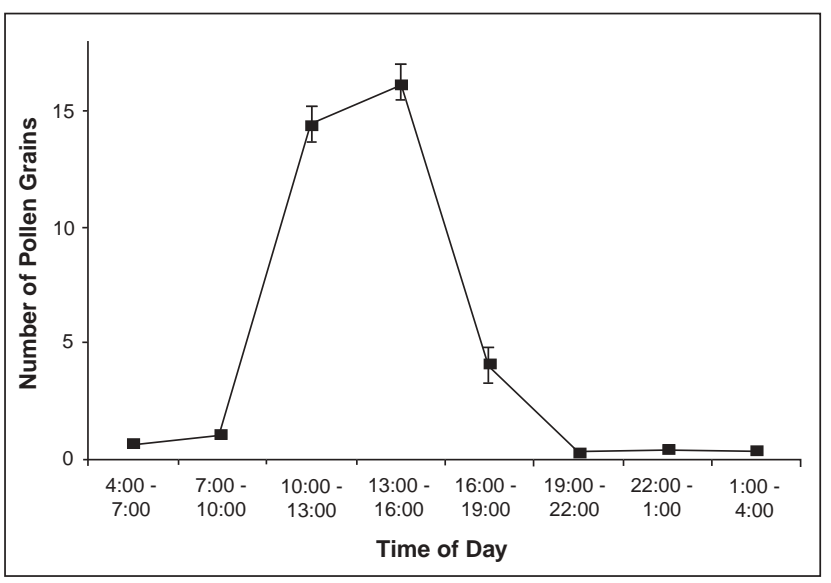

ested in studying wind pollination on a practical level. This experiment would be most appropriate for upper level biology students, based on the difficulty of the set-up and analysis of the results. The flexibility of the protocol, locations and conditions, means that an experiment can easily be applied to a number of different plant species from trees to herbs. The system can also be deployed on urban buildings to monitor potentially allergenic pollens in the cityscape. This allows for a wide range of information to be obtained from the experimental use of these pollen traps.

\section{References}

A ston T J (1987) Plant pollinator interactions - A rich area for study. Journal of Biological Education 21, 267-274.

Barton J E and Dracup M (2000) Genetically modified crops and the environment. A gronomy Journal 92, 797-803.

Bhalla P L, Swoboda I and Singh M B (1999) Antisense-mediated silencing of a gene encoding a major ryegrass pollen allergen. Proceedings of the $\mathrm{N}$ ational A cademy of Sciences of the $U$ nited States of A merica 96, 11676-11680.

Claypoole S T and Slesnick I L (1983) The beauty and biology of pollen. A merican Biology Teacher 45, 366-370.

Dafni A, Pacini E and N epi M (2005) Pollen and stigma biology. In: Practical Pollination Biology, eds D afni A, Kevan P G and H usband B C pp83-146. Cambridge, Canada. Enviroquest Ltd.

Dale P J, Clarke B and Fontes E M G (2002) Potential for the environmental impact of transgenic crops. N ature Biotechnology 20, 567-574

DiGiovanni F, Kevan P G and Arnold J (1996) Lower planetary boundary layer profiles of atmospheric conifer pollen above a seed orchard in northern O ntario. Forestry C hronicle 72, 166-169.

Kevan P G (2005) Pollination by wind (anemophily). In: Practical Pollination Biology, eds Dafni A, Kevan P G and Husband B C pp439-464. Cambridge, Canada. Enviroquest Ltd.

Losey J E, Rayor L S and C arter M E (1999) Transgenic pollen harms monarch larvae. $N$ ature 399, 214.

Marquez J, Seoane-Camba J A and Suarez-Cervera M (2002) Allergenic and antigenic proteins released in the apertural sporoderm during the activation process in grass pollen grains. European Journal of C ell Biology 81, 107-115.

Snetselaar K M , Carfioli M A and Cordisco K M (2001) Pollination can protect maize ovaries from infection by $U$ stilago maydis, the corn smut fungus. Canadian Journal of Botany 79, 1390-1399.

Tse H L H and Chan G Y S (2001) Pollen germination - a challenging and educational experiment. J ournal of Biological Education 35, 148-151.

\footnotetext{
Peter G Kevan (corresponding author) is a professor in the Department of Environmental Biology, U niversity of G uelph, G uelph, 0 ntario, Canada N1G 2W1. Tel: + 1 519-824-4120 Ext 52479. Fax: +1 519-837-0442. Email: pkevan@uoguelph.ca. Franco D iG iovanni is a research scientist at AirZO ne Inc, M ississauga, $C$ anada. Email: fdi-giovanni@airzoneone.com. Rong H $\mathrm{Ho}$ is a retired scientist. $\mathrm{H}$ isatomo Taki is a graduate student in the Department of Environmental Biology, University of Guelph. Email: htaki@uoguelph.ca. Kristyn A Ferguson is a graduate student in the Department of Environment and Resource Studies, University of Waterloo, C anada. Email: k2fergus@fes.uwaterloo.ca. Agata K. Pawlowski is a research technician in the D epartment of Environmental Biology, U niversity of G uelph. Email: pawlowsk@uoguelph.ca
} 